\title{
Enhanced SERS Activity Induced by the Ag Rod-Fe304 Nanocomposite with Different Applied Magnetic Field
}

\author{
Chunzhen Fan ${ }^{1, a^{*}}$ and Shuangmei Zhu ${ }^{2, b}$ \\ ${ }^{1}$ School of Physical Science and Engineering, Zhengzhou University, Zhengzhou 450052, China \\ ${ }^{2}$ College of Science, Henan Institute of Engineering, Zhengzhou, 451191, China

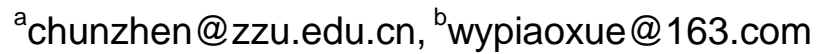

Keywords: SERS; $\mathrm{Fe}_{3} \mathrm{O}_{4}$ nanoparticles; Magnetic field; Optical resonance

\begin{abstract}
Fe}_{3} \mathrm{O}_{4}$ magnetic nanoparticles (MNPs) were synthesized by a co-precipitation method using the solution of ferrous/ferric mixed salt, and then an alkali solution is dropped into the mixed metal solution. With the seed mediated growth method, $\mathrm{Ag}$ nanorods were also obtained. SERS-active substrate composed of the $\mathrm{Ag}$ rod and $\mathrm{Fe}_{3} \mathrm{O}_{4}$ nanocomposites were fabricated with an applied magnetic field, which was ranging from 1450, 2500 and 3500 gauss. With the probed crystal violet (CV) molecule, SERS spectra were carried out. Our experimental results display that enhanced Raman signal can be obtained with the increased magnitude of magnetic field. It happens due to the enhanced electromagnetic field, which was caused by coupling effect of the local Plasmon resonance and the propagating Plasmon resonance. The magnetic nanoparticle forms uniform array under the influence of the external magnetic field. These uniformly arrayed distributions thereby enhance the local resonance of the above deposited Ag rod nanoparticles. Our proposed metallic-magnetic nanocomposites enable us a much free method to get the enhanced SERS effect, which can be used in the optical sensing, single molecule SERS, and bio-world applications.
\end{abstract}

\section{Introduction}

Since the discovery of the surface enhanced Raman scattering originated from electromagnetic enhancement by Jeanmarie and Van Duyne [1] and chemical enhancement by Albrecht and Creighton [2] in 1977 respectively. Great efforts have been devoted to study the SERS effect, owing to its high specify, high sensitivity and unique identifies molecules, which is a much valuable tool for analyzing mixtures $[3,4]$. It can provide the detailed finger print of the material information at single molecule level and the enhancement factor [5]. The key factor to obtain the repeatable, stable, and high sensitive SERS signal is the design of SERS substrate. There is a large variety of SERS substrates include colloids, rough electrodes, and metal island films, aggregation of metal nanoparticles [6-12]. While to get the repeatable SERS signal, external field can renders us another degree to fabricate the SERS substrate. As the basic requirement for the sensitive SERS detection, an ideal substrate must guarantee a high enhancement effect and a uniform response [13]. Both theoretically and experimentally develop the robust SERS substrate embody the enhanced electromagnetic field [14]. With the metallic nanoparticles, the existence of collective excitations around the metallic nanoparticles was localized surface Plasmon resonance, which is actually the eigenmodes of a photon-driven coherent oscillation of electrons in metal nanoparticles. Light coupling to surface Plasmon can result in large localized field enhancement [15]. As for the $\mathrm{Fe} 3 \mathrm{O} 4$ magnetic nanoparticles, its superparamagnetic characteristics render it forming uniform array under the influence of the external magnetic field $[16,17]$. Combining the optical and magnetic response, we have already investigated the realization of high sensitive SERS substrates with one-pot fabrication of $\mathrm{Ag}-\mathrm{Fe} 3 \mathrm{O} 4$ nanocomposites, where the SERS substrate has a good reproducibility with low standard deviation [18].

In this work, we investigate the external magnetic field tunable SERS substrate based on the Ag nanorod and $\mathrm{Fe} 3 \mathrm{O} 4$ nanocomposites. With the probe $\mathrm{CV}$ molecule, the detection of SERS spectra 
were carried out when the external magnetic field was varying from 1450, 2500 to 3500 gauss. Incorporating the Plasmon resonant effect of the silver nanoparticles and the magnetic field controllable effect, Raman spectra were carried out to test the controllability and reproducibility of our prepared SERS substrate.

\section{Experimental Section}

Materials. Silver nitrate $\left(\mathrm{AgNO}_{3}\right)$, ascorbic acid $\left(\mathrm{C}_{6} \mathrm{H}_{8} \mathrm{O}_{6}\right)$, cetyltrimethylammonium bromide $\left(\mathrm{C}_{19} \mathrm{H}_{42} \mathrm{BrN}, \mathrm{CTAB}\right)$, sodium hydroxide $(\mathrm{NaOH})$, tri-sodium citrate $\left(\mathrm{C}_{6} \mathrm{H}_{5} \mathrm{Na}_{3} \mathrm{O}_{7}\right)$, sodium borohydride $\left(\mathrm{NaBH}_{4}\right)$, ferrous chloride $\left(\mathrm{FeCl}_{2} \bullet 4 \mathrm{H}_{2} \mathrm{O}\right)$, ferric chloride hexahydrate $\left(\mathrm{FeCl}_{3} \bullet 6 \mathrm{H}_{2} \mathrm{O}\right)$, crystal violet $(\mathrm{CV})$, sodium citratedihydrate $\left(\mathrm{Na}_{3} \mathrm{C}_{6} \mathrm{H}_{5} \mathrm{O}_{7} .2 \mathrm{H}_{2} \mathrm{O}, 99 \%\right)$ were purchased from Zhengzhou Fuyou Chemical (China). All chemical reagents were used without further processing. Aqueous solutions were prepared using distilled de-ionized water.

Characterization. Raman spectra were collected at room temperature by Renishaw-2000 Raman spectrometer with $532 \mathrm{~nm}$ laser as an excitation source. The laser is focused onto the samples from the top by an objective (50×, NA, 0.55).

Experimental Procedures. Magnetite nanoparticles were prepared by coprecipitation reaction of ferrous $\left(\mathrm{Fe}^{2+}\right)$ and ferric $\left(\mathrm{Fe}^{3+}\right)$ ions with the presence of $\mathrm{NaOH}$ or $\mathrm{NH}_{3} \cdot \mathrm{H}_{2} \mathrm{O}$ [17]. Silver nanorods were synthesized with seed-mediated growth method, following the procedures described in Ref [19-21].

Preparation of Ag rod and $\mathrm{Fe}_{3} \mathrm{O}_{4}$ Nanoparticles SERS Substrate. The magnet was placed right below the clean glass slip, then $10 \mathrm{uL} \mathrm{Fe}_{3} \mathrm{O}_{4}$ were deposited. After the evaporation, $\mathrm{Ag}$ rod solution was dropped. Finally, different concentration of $\mathrm{CV}$ molecule absorb on the $\mathrm{Ag}$ and $\mathrm{Fe}_{3} \mathrm{O}_{4}$ nanoparticles. Let the as prepared SERS substrate dry under ambient conditions. All samples were used immediately after preparation for SERS measurements.

\section{Results and Discussions}

Raman spectra of the $10^{-6} \mathrm{M} \mathrm{CV}$ molecule absorbed on the $\mathrm{Ag}$ rod and $\mathrm{Fe}_{3} \mathrm{O}_{4}$ nanocomposites were measured with an excitation wavelength of $532 \mathrm{~nm}$. The results are shown in Fig. 1. When the external magnetic filed was initiated, SERS effect can be clearly observed in Fig. 1(b). The enhanced Raman signal was originated from the enhanced local electric fields arising around the metallic Ag rod nanoparticles. It indicates an electromagnetic coupling of the localized surface plasmon around the surface of the $\mathrm{Ag}$ rod nanoparticles induced by the magnetic aggregations of the metallic nanoparticles. The assignments of the Raman modes can be found in the Ref. [18]. The results indicate that external magnetic field causes an enhanced SERS optical activity. In details, tunable magnetic field was initiated on the SERS substrate in Fig. 2, where the external magnetic field is ranging from 1450, 2500, 3500 gauss respectively. It can be clearly observed that enhanced signal can be detected. It happens due to the ordered arrayed magnetic nanoparticle under the influence of the external magnetic field. These uniformly arrayed distributions thereby enhance the local resonance of the above deposited Ag rod nanoparticles. Thus, the occurrence of two plasmon resonances exists, namely, the local surface plasmon resonance and propagating plasmon resonant along the uniform distribution of the metallic nanoparticles array. The results illustrate that enhanced Raman signal can be detected with the increased magnetic field, indicating more and more magnetic nanoparticles form uniform array under the influence of the external magnetic field. 


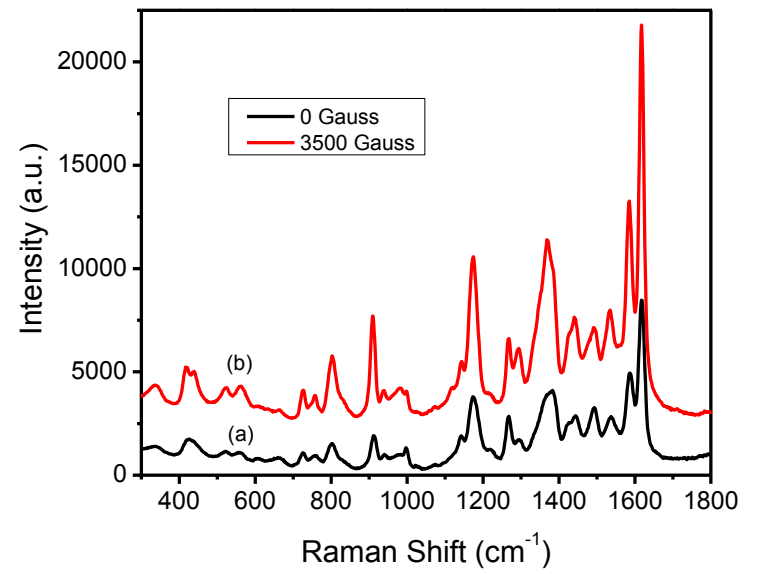

Figure 1. Raman spectra of $10^{-6} \mathrm{M} \mathrm{CV}$ molecule of $\mathrm{Ag}$ rod and rod and $\mathrm{Fe}_{3} \mathrm{O}_{4}$ nanocomposite without (a) of the and with (b) applied magnetic field. gauss).

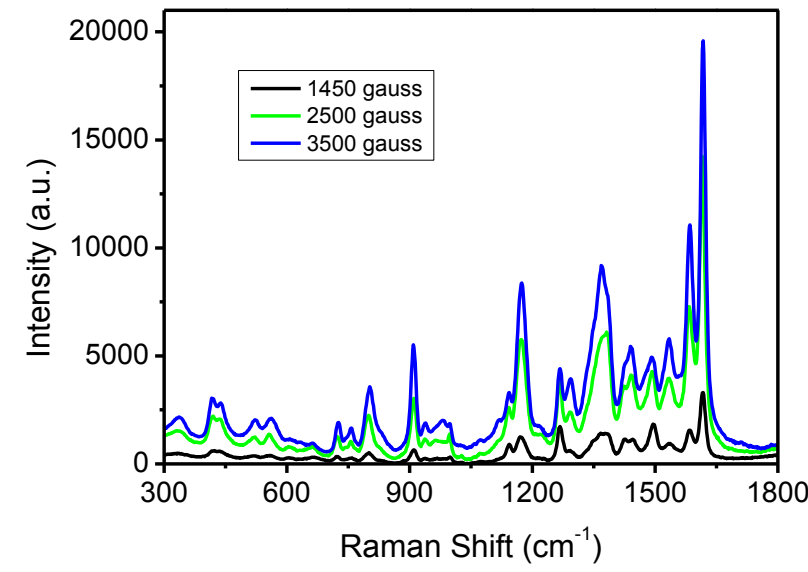

Figure 2. Raman spectra of CV molecule with $\mathrm{Ag}$ $\mathrm{Fe}_{3} \mathrm{O}_{4}$ nanocomposite with different magnitude applied magnetic field (a.1450, b. 2500, c. 3500

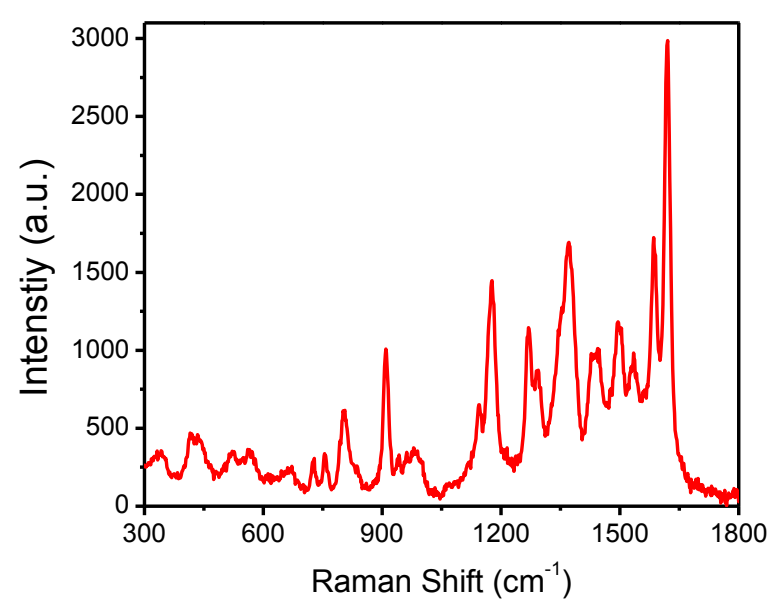

Figure 3. Raman spectra of $10^{-7} \mathrm{M} \mathrm{CV}$ molecule with $\mathrm{Ag}$ rod and $\mathrm{Fe}_{3} \mathrm{O}_{4}$ nanocomposite with 3500 gauss magnetic field.

Under the influence of the 3500 gauss magnetic field, we have also investigated the $10^{-7} \mathrm{M} \mathrm{CV}$ molecule with $\mathrm{Fe}_{3} \mathrm{O}_{4}$ and $\mathrm{Ag}$ nanocomposite SERS substrate, where each Raman signal of the CV molecule can be clearly identified in Fig. 3. It verified the identifications capability of our prepared SERS substrate. Thus, our magnetic field controllable substrate can be employed as a perfect SERS probe for molecule detection at lower concentrations.

\section{Conclusions}

$\mathrm{Ag}$ rod nanoparticles was synthesized with the seed mediated growth method and the $\mathrm{Fe}_{3} \mathrm{O}_{4}$ nanoparticles were obtained by using the coprecipitation methods. With the combination of the Ag nanorod and $\mathrm{Fe}_{3} \mathrm{O}_{4}$ nanoparticles, we studied the uniformity and spectral reproducibility of the SERS substrate with controllable magnetic field. With the initiation of the different magnitude of the external magnetic field, the experimental results show that enhanced Raman signal of the CV molecule was obtained due to the coupling of the plasmon band of the irradiated Ag nanoparticle, which given rise to the enhanced SERS effects. Light coupling to surface plasmons of Ag rod nanoparticle can result in large localized field enhancement. Moreover, the uniform array of the 
magnetic nanoparticle hereby enhances the propagating plasmon resonance effect of the Ag nanorod nanoparticles. The experimental results demonstrate good reproducibility and enhancement. It indicates that our prepared SERS substrate is a good candidate for the chemical and biological sensing application.

\section{Acknowledgements}

This work is supported by the Key science and technology research project of Henan Province (162102210164), by the Foundation of Henan Educational Committee (17A140002).

\section{References}

[1] D. L. Jeanmaire, R. P. Van Duyne, J. Electroanal. Chem. 84, 1-20 (1977).

[2] M.G. Albrecht, J.A. Creighton, J. Am. Chem. Soc. 99, 5215-5217 (1977).

[3] J.A. Dieringer, A.D. McFarland, N.C. Shah, D.A. Stuart, A.V. Whitney, C.R. Yonzon, M.A. Young, X. Zhang, R.P. Van Duyne, Faraday Discuss. 132, 9-26 (2006).

[4] K.C. Bantz, A.F. Meyer, N.J. Wittenberg, H. Im, O. Kurtuluş, S.H. Lee, N.C. Lindquist, S.H. Oh, C.L. Haynes, Phys. Chem. Chem. Phys. 13, 11551-67(2011).

[5] K. Kneipp, H. Kneipp, I. Itzkan, R.R. Dasari, M.S. Feld, Chem. Rev. 99, 2957-2975 (1999).

[6] C.J. Murphy, T.K. Sau, A.M. Gole, C.J. Orendorff, J.X. Gao, L.F. Gou, S.E. Hunyadi, T. Li, Phys. Chem. B 109, 13857-13870 (2005).

[7] Q. Liao, C. Mu, D.S. Xu, X. C. Ai, J. N. Yao, J.P. Zhang, Langmuir. 25, 4708-4714 (2009).

[8] Y. Yang, L. Xiong, J. Shi, M. Nogami, Nanotechnology, 17, 2670-2674 (2006).

[9] G.K. Wei, J.L. Wang, Y. Chen, Beilstein J. Nanotechnol. 6, 686-696 (2015).

[10] M.K. Hossain, Y. Kitahama, G.G. Huang, X. Han, Y. Ozaki, Anal. Bioanal. Chem. 394, 1747-1760 (2009).

[11] S. Lal, N.K. Grady, J. Kundu, C.S. Levin, J.B. Lassiter, N.J. Halas, Chem. Soc. Rev. 37, 898-911 (2008).

[12] Y.Q. Wen, W.Q. Wang, Z.L. Zhang, L.P. Xu, H.W. Du, X.J. Zhang, Y.L. Song, Nanoscale ,5, 523-526 (2013).

[13]L.P. Du, X.J. Zhang, T. Mei, and X.C. Yuan, Opt. Expr. 18, 1959-1965 (2010).

[14] R.F. Aroca, R.A. Alvarez-Puebla, N. Pieczonka, S. Sanchez-Cortez, J.V. Garcia-Ramos, Adv. Colloid Interf. 116, 45-61 (2005).

[15] M. Schwind, B. Kasemo, I. Zorić, Nano Lett. 13, 1743-1750 (2013).

[16] J. Gao, H. Gu, B. Xu, Accounts Che. Res. 42, 1097-1107 (2009).

[17] L.H. Reddy, J.L. Arias, J. Nicolas, P. Couvreur, Chem. Rev. 112, 5818-5878 (2012).

[18] S.M. Zhu, C.Z. Fan, J.Q. Wang, J.N. He, E.J. Liang, M.J. Chao, J. Colloid Interf. 438, 116-121 (2015).

[19] N.R. Jana, L. Gearheart, C.J. Murphy, Adv. Mater. 13, 1389-1393 (2001).

[20] N.R. Jana, L. Gearheart, C. J. Murphy, J. Phys. Chem. B 105, 4065-4067 (2001).

[21] G.J. Lee, S. Shin, Y.C. Kim, S.G. Oh, Mater. Chem. Phys. 84, 197-204 (2004) 